\title{
Prognostische pulmonale und thorakale Befunde bei Patienten mit instabilen Verletzungen der Brustwirbelsäule
}

\author{
Prognostic Pulmonary and Thoracic Findings in Patients with Unstable Injuries \\ of the Thoracic Spine
}

Autoren

Institute
S. P. Lemburg' ${ }^{1}$, T. M. Frangen ${ }^{2}$, H. Knoop ${ }^{3}$, V. Nicolas' ${ }^{1}$, C. M. Heyer ${ }^{1}$

Die Institutsangaben sind am Ende des Beitrags gelistet. eingereicht 28.10.2010 akzeptiert nach Revision 25.1.2011

\section{Bibliografie}

DOI http://dx.doi.org/ 10.1055/s-0030-1256218

Online-Publikation: 2. 3. 2011

Pneumologie 2011; 65:

412-418 ๔ Georg Thieme

Verlag KG Stuttgart · New York ISSN 0934-8387

\section{Korrespondenzadresse}

PD Dr. med.

\section{Christoph M. Heyer}

Institut für Diagnostische Radiologie, Interventionelle Radiologie und Nuklearmedizin Berufgenossenschaftliches Universitätsklinikum Bergmannsheil GmbH Ruhr-Universität Bochum Bürkle-de-la-Camp Platz 1 44789 Bochum christoph.heyer@rub.de

\section{Zusammenfassung \\ V}

Hintergrund: Instabile BWS-Verletzungen können ein erhebliches Trauma der intrathorakalen Organe nach sich ziehen. Ziel der Studie war die systematische Analyse der prognostischen Wertigkeit von CT-Befunden an Lunge, Mediastinum und Thoraxwand im Hinblick auf die Mortalität. Methode: Mehrfachverletzte bzw. polytraumatisierte Patienten mit instabiler BWS-Fraktur und initialer thorakaler CT-Diagnostik wurden retrospektiv im Zeitraum April 2004 bis Mai 2007 erfasst. Folgende Bildbefunde wurden hinsichtlich prognostischer Wertigkeit für die Mortalität analysiert: Verletzungen von Lunge/Pleura (Pneumothorax, Ergüsse, Lungenkontusionen/-lazerationen, Atelektasen, Hilusverletzungen), Mediastinum (Gefäßverletzungen, Blutungen, Zwerchfellverletzungen) und Thoraxwand (Prellmarken, Weichteilemphysem, Rippenfrakturen) und Drainagenpflichtigkeit. Das Signifikanzniveau wurde bei $p=0,05$ festgelegt.

Patienten: Von 33 Patienten (Durchschnittsalter 43,5 $\pm 20,1$ Jahre [Spannweite 14-83 Jahre]; 25 männlich [76\%]) verstarben 7 (21\%), wobei Frauen hochsignifikant häufiger betroffen waren $(p<0,001)$. Das Patientenalter zeigte keine signifikante Assoziation mit der Mortalität.

Ergebnisse: Verstorbene zeigten einen signifikant höheren Lungenkontusions-Score (Median der Verstorbenen: 4,0 [0-12] vs Überlebende: 1,0 $[0-10] ; p=0,016)$. Folgende Bildbefunde waren signifikant mit der Mortalität assoziiert: Thorakale Prellmarken mit Weichteilemphysem $>90^{\circ}$ des thorakalen Querschnitts (Sensitivität [SE]: 43\%, Spezifität [SP]: $100 \%$, positiver Vorhersagewert [PPW]: 100\%, negativer Vorhersagewert [NPW]: $87 \%$, Gesamtaussagekraft [OA]: $88 \% ; p=0,006$ ), Rippenfrakturen (SE: 71\%, SP: 81\%, PPW: 50\%, NPW: 91\%, OA; 79\%; $p=0,016)$, mediastinale Hämatome (SE: 71\%, SP: 77\%, PPW: 45\%, NPW: $91 \%$,

\section{Abstract \\ $\nabla$}

Background: Instable fractures of the thoracic spine imply a substantial trauma to the chest. The aim of this study was to undertake a systematic analysis of the prognostic impact of CT findings of the chest wall, mediastinum, lungs, and pleural space on the mortality rate.

Method: All multiple injury patients with instable fractures of the thoracic spine and initial CT scans treated in our clinic from April 2004 to May 2007 were eligible. The following variables were evaluated for their prognostic power: injury to the lungs (pneumothorax, effusion, lung contusions/lacerations, atelectasis), mediastinum (vessel dissection/rupture, bleeding, diaphragmatic rupture), chest wall (bruises, soft tissue emphysema, rib fractures), and need for pre-diagnostic tube thoracostomy. The significance level was set to $p=0.05$.

Patients: Of a total of 33 patients (mean age: $43.5 \pm 20.1$ years [range $14-83$ years]; 25 -male [76\%]), seven patients $(21 \%)$ died with women being significantly more affected $(p<0.001)$. Mortality rate was not influenced by patient age.

Results: Non-survivors displayed significantly higher median lung contusion score values compared to survivors (4.0 [0-12] versus $1.0[0-10]$; $p=0.016$ ). The following variables revealed a significant association with the mortality rate: chest wall bruises with soft tissue emphysema $>90^{\circ}$ of the thoracic circumference (sensitivity [SE]: $43 \%$, specificity [SP]: $100 \%$, positive predictive value [PPV]: $100 \%$, negative predictive value [NPV]: $87 \%$, overall accuracy [OA]: $88 \% ; p=0.006$ ), rib fractures (SE: 71\%, SP: $81 \%$, PPW: 50\%, NPW: 91\%, OA: 79\%; $p=0.016)$, mediastinal haematoma (SE: 71\%, SP: 77\%, PPW: 45\%, NPW: 91\%, OA: $76 \% ; p=0.027$ ), and bilateral pneumothoraces (SE: 29\%, SP: $100 \%$, PPW: $100 \%$, NPW: $84 \%$, OA: $85 \% ; p=0.040$ ). 
OA: 76\%; $p=0,027)$ und bilaterale Pneumothoraces von $1-3 \mathrm{~cm}$ Saumbreite (SE: 29\%, SP: 100\%, PPW: 100\%, NPW: $84 \%$, OA: $85 \% ; p=0,040)$.

Schlussfolgerungen: Bei instabiler BWS-Fraktur zeigen einzelne thorakale Bildbefunde eine signifikante Assoziation mit der Mortalität. Die CT-Diagnostik muss damit sowohl der BWS-Abklärung als auch der Evaluation von Thorax und Lunge Rechnung tragen.
Conclusion: Multiply injured patients with instable fractures of the thoracic spine display CT findings indicative of non-survival. Image acquisition and interpretation focusing solely on the spine should be avoided to ensure the detection of prognostic injury patterns to the lungs.

\section{Abkürzungen}

BWK Brustwirbelkörper

BWS Brustwirbelsäule

CT Computertomografie

HU Hounsfield-Einheiten

LWK Lendenwirbelkörper

MDCT Multidetektor-Computertomografie

\section{Einleitung}

Verkehrsunfälle stellen bei jüngeren Patienten eine der häufigsten Todesursachen dar . Bei etwa $25 \%$ der traumatisch bedingten Todesfälle liegen zudem Thoraxtraumen vor [2], welche neben der initialen und stationären Versorgung der Patienten auch deren Prognose stark beeinflussen können. Eine zeiteffiziente Diagnostik sowie effektive Therapie des polytraumatisierten Patienten ist gemäß dem Konzept der „golden hour of trauma care“ [3] entscheidend, da eine hier nicht oder unzureichend durchgeführte Primärdiagnostik für bis zu 36\% der vermeidbaren Todesfälle verantwortlich gemacht werden kann $[4,5]$. Neben der Notwendigkeit der klinischen Einschätzung nach diversen Trauma-ScoreSystemen (Revised Trauma Score, Organ Injury Scale, Abbreviated Injury Scale, Injury Severity Score) kommt damit der initialen Bildgebung eine Schlüsselrolle zu. Während die CT-Diagnostik vor Einführung der Multidetektor-Computertomografen (MDCT) aufgrund der aufwendigen Akquisition und Berechnung des Bildmateriales einer gezielten Abklärung einzelner Befunde vorbehalten war, ist mit neuen CT-Scannern die Erfassung großvolumiger Körperabschnitte mit Berechnung dünnschichtiger, annähernd isotroper Datensätze deutlich schneller möglich. Aus diesen Volumendatensätzen können neben multidimensionalen Rekonstruktionen singulärer Organe in sämtlichen Raumebenen auch unterschiedliche Bereiche des Körpers mittels größenvariabler Ausschnitte in kürzester Zeit und hoher Qualität berechnet werden. Dem Untersucher steht damit ein Werkzeug zur Verfügung, welches ein hohes Maß an Informationen in kurzer Zeit bereithält. Der trotz höherer Strahlenexposition der Untersuchten [6] zunehmende Einsatz der MDCT in der Versorgung von Traumatisierten [7-10] findet hierdurch - neben der höheren Aussagekraft bezüglich der Wirbelsäule im Vergleich zur konventionell-radiologischen Diagnostik [11 -15] - eine Erklärung. Darüber hinaus ist bekannt, dass bei einer instabilen Verletzung der Brustwirbelsäule (BWS) angesichts der hierfür nötigen Krafteinwirkung auf das muskuloskelettale Ensemble der Wirbelsäule ein erhebliches Thoraxtrauma stattgefunden haben kann [16]. Die hieraus resultierenden Verletzungen der Lungen, des Mediastinums und der Thoraxwand können sowohl das Management als auch die Patientenprognose erheblich beeinflussen [2]: Pulmonale Komplikationen sind die führende Ursache für die Morbidität und Mortalität spinal verletzter Patienten [17,18]. Die
Versorgung dieser komplex verletzten Patienten kann eine frühzeitige Einbeziehung und engen fachübergreifenden Austausch aller Disziplinen erfordern, da die konservative begleitende Therapie einen erheblichen - im Fall des Thoraxverletzten entscheidenden - Einfluss auf die Prognose hat. Der internistischen bzw. pneumologischen Betreuung und Beurteilung dieser Patientengruppe kommt daher oft ebenso eine Bedeutung zu, sodass ein rasches Erkennen prognostischer CT-Bildbefunde bei BWS- bzw. konsekutiv Thorax-Verletzten auch für primär nicht-operative Disziplinen von Wert ist. Als Haus der Maximalversorgung mit Schwerpunkt für Unfall- und Wirbelsäulenchirurgie versorgt das Berufsgenossenschaftliche Universitätsklinikum Bergmannsheil Bochum regelmäßig polytraumatisierte bzw. mehrfach verletzte Patienten mit Wirbelsäulentraumata. Eine Evaluation der pulmonalen und thorakalen Begleitbefunde dieser Patientengruppe erschien damit sinnvoll.

Ziel dieser Studie war die systematische retrospektive Analyse von Bildbefunden an Lunge, Mediastinum und Thoraxwand, basierend auf der initialen thorakalen MDCT-Diagnostik bei polytraumatisierten bzw. mehrfach-verletzten Patienten mit instabiler BWS-Verletzung im Hinblick auf ihre prognostische Wertigkeit.

\section{Material und Methoden \\ $\nabla$}

Auf ein Votum der Ethikkommission wurde angesichts des rein retrospektiven Studiencharakters auf Grundlage der Bilddaten und Aktenlage verzichtet.

\section{Studienpopulation und Bilddaten}

Über das radiologische Informationssystem (RIS) erfolgte zunächst eine Identifikation aller in unserem Klinikum initial mittels Polytrauma-CT diagnostizierten Patienten im Zeitraum 01. 04. 2004 bis 31.05. 2007. Einschlusskriterien waren die initiale Einschätzung als Polytrauma-Patient, der Nachweis einer instabilen BWS-Verletzung, die Verfügbarkeit einer innerhalb der ersten 48 Stunden nach Trauma angefertigten thorakalen CT-Diagnostik inklusive Darstellung der BWS und das Vorliegen aller Bilddaten mit sagittalen und koronaren Rekonstruktionen. Die Studienpopulation umfasste neben polytraumatisierten auch mehrfach-verletzte Patienten mit instabiler BWS-Verletzung. Neben Patienten mit rein thorakalen Wirbelsäulenverletzungen wurden auch Verletzte mit Frakturen des thorakolumbalen Überganges bis auf Höhe LWK 2 einbezogen, da oft eine gleiche anatomische Höhe der dorsalen Rezessus, der 11./12. Rippen bzw. der posterobasalen und -lateralen Unterlappensegmente mit den LWK1 / 2 gegeben ist und infolgedessen Verletzungen des thorakolumbalen Überganges mit thorakalen und pulmonalen Verletzungen resultieren können. Als instabil wurden nach dem schriftlichen radiologischen Befund alle Frakturen mit Beteiligung von mindestens zwei Wirbelsäulen-Kompartimenten (nach 
dem Modell von Denis) und/oder begleitender Rotationsverletzung definiert. Im Falle einer fraglichen Stabilität nach radiologischem Befund wurden diese Frakturen in interdisziplinärer Konsensusentscheidung erneut anhand des Bildmateriales als stabil oder instabil bewertet. Darüber hinaus wurden die Patientenakten im Hinblick auf Patientencharakteristika (Alter, Geschlecht), Dauer des Krankenhausaufenthaltes und die klinische Zielvariable Mortalität unabhängig von der Todesursache ausgewertet. Für die in die Studiengruppe aufgenommenen Patienten lag eine thorakale CT bzw. eine Polytrauma-CT am Unfall- oder Folgetag mit folgenden technischen Parametern vor: 16-Zeilen-Scanner (SOMATOM Sensation $16^{\circledR}$, Siemens, Erlangen), Untersuchung nach intravenöser Injektion von 80-100 ml iodhaltigem, wasserlöslichem Kontrastmittel (Flussrate $3 \mathrm{ml} / \mathrm{s}$; Delay $30 \mathrm{~s}$ ) unter Erfassung der gesamten BWS bis auf Höhe LWK2, Kollimation $1,5 \mathrm{~mm}$, axiale Schichtdicke $6,0 \mathrm{~mm}$, Rekonstruktionen mit weichem/hartem Kernel (B30/B60) und Ganzkörper-Field-of-View, Dokumentation im Weichteil-/Lungenfenster. Aus dem so gewonnenen Datensatz erfolgten zudem axiale, coronare und sagittale Rekonstruktionen der BWS. Sämtliche Bildanalysen wurden am Monitor durchgeführt.

\section{Datenauswertung}

Die resultierenden Bilddaten wurden durch zwei erfahrene Radiologen mit Facharzt-Status im Konsensus evaluiert. Die nachfolgend aufgeführten pulmonalen und thorakalen Befunde wurden mittels eines standardisierten und an der Organ Injury Scale (OIS) der American Association for the Surgery of Trauma (AAST) orientierten Bogens dokumentiert:

- Bezüglich des Thorax wurden Verletzungen des Weichteilmantels zunächst allgemein erfasst und dann getrennt für Prellmarken mit jeweilig begleitenden Veränderungen der Muskulatur und/oder Lufteinsprengungen/Fremdkörpern nach Größe aufgeführt $\left(<45^{\circ} / 45-90^{\circ} />90^{\circ}\right.$ des thorakalen Querschnitts auf Höhe der größten Ausdehnung). Als Prellmarke wurden Dichteanhebungen des subkutanen Fettgewebes mit mindestens flüssigkeits-isodensen Dichtewerten definiert. Als Trauma der Muskulatur galten asymmetrische, nicht der Muskelanatomie folgende Unterschiede des Muskelvolumens mit Unschärfe bzw. Maskierung der Fettgewebsräume.

- Verletzungen des Thoraxskeletts wurden insgesamt und getrennt für Rippen nach Anzahl (uni-/bilaterale Rippenfrakturen nach Anzahl (1 - 3/>3) und Form (Stück-/Trümmerfraktur, mit Einspießung/Dislokation), für Sternumfrakturen (nicht-disloziert/-disloziert/Trümmerfraktur) und für Clavicula- und Scapulafrakturen (nicht-disloziert/-disloziert/ Trümmerfraktur/beidseitig) evaluiert.

- Die BWS-Verletzungen wurden im Hinblick auf Höhenlokalisation, Anzahl der Frakturen und ggf. vorhandene Quer- und Dornfortsatzfrakturen untersucht.

- Als Verletzungen der großen Gefäße wurden Dissektionen/ Rupturen der Aorta, der supraaortalen Arterien oder der V. cava superior definiert. Darüber hinaus wurden mediastinale Hämatome (bis oder über $5 \mathrm{~cm}$ Größe) unabhängig von ihrer Genese, aktive mediastinale Blutungen aus extraaortalen Gefäßen, Perikardergüsse (bis $1 \mathrm{~cm}$ Größe, $1-3 \mathrm{~cm}$, größer $3 \mathrm{~cm}$ ) und traumatische Zwerchfellrupturen (rechts/links, mit/ohne Enterothorax) dokumentiert.

- An den Lungen wurden dorsale, lagebedingte Minderbelüftungen und sonstige Atelektasen erfasst (Anzahl der Segmente, uni-/bilateral $<1$ Segment $/<1$ Lappen $/<1$ Lunge, Total- atelektase). Zudem wurden Pneumothoraces nach Seite und Ausdehnung (uni-/bilateral $<1 \mathrm{~cm}, 1-3 \mathrm{~cm},>3 \mathrm{~cm}$, Spannungspneumothorax) und Lungenkontusionen/Einblutungen nach Seite (uni-/bilateral), Ausdehnung (<25\%, $25-50 \%$, $>50 \%$ der Fläche) und Lokalisation (Ober-, Mittel- und Untergeschoss) bewertet. Als Kontusion/Einblutung wurde eine nicht einer Atelektase entsprechende, nicht anatomisch geformte Konsolidierung mit milchglasartiger oder unscharfer Begrenzung definiert. Als Obergeschoss galt die Ebene auf Höhe des Oberrandes des Arcus aortae, als Mittelgeschoss die Ebene auf Höhe des Abgangs des ML-Bronchuś und als Untergeschoss die Ebene auf Höhe der Zwerchfell-Kuppeln. Als Kontusionsscore erfolgte eine Scoreaddition mit folgenden Scorewerten: Bei Kontusion/Einblutung $<25 \%=1$, $25-50 \%=2,>50 \%=3$, getrennt nach Seiten und Höhe (minimaler Scorewert: 0, maximaler Wert: 18). Das Vorliegen von unilateralen Hilusverletzungen oder uni-/bilateralen Lungenlazerationen, definiert als freie nicht anatomische Lufteinsprengung in das Lungenparenchym in einem konsolidierten Lungenanteil (exklusive Bulla/Zyste), wurden erfasst. Darüber hinaus erfolgte eine Dokumentation ggf. einliegender Thoraxdrainagen.

Als Zielvariable wurde die Mortalität dokumentiert.

\section{Statistische Auswertung}

Die statistische Auswertung erfolgte an einem Standard-PC mittels SPSS ${ }^{\circledR}$ for Windows V15.0 (SPSS Inc., Chicago, IL, U.S.A.). Die qualitativ definierten Bildbefunde (kategorische Variablen) wurden im Hinblick auf die Zielvariable mittels Chi-Quadrat-Test evaluiert. Darüber hinaus erfolgte eine multivariate Analyse der explorativ identifizierten Faktoren. Für den Scorewert für Kontusionen/Einblutungen (numerische Variable) wurde ein MannWhitney-Test angewandt. Der Zusammenhang zwischen Patientenalter und Mortalität wurde mittels student's t-Test evaluiert. Das Signifikanzniveau wurde auf $5 \%$ festgelegt.

\section{Ergebnisse \\ $\nabla$}

\section{Studienpopulation}

Im Studienzeitraum wurden 102 polytraumatisierte bzw. mehrfach-verletzte Patienten mit BWS-Fraktur behandelt. Nach radiologischem Befund und Konsensuseinschätzung mussten 69 dieser Patienten (68\%) wegen nicht verfügbarer oder unvollständiger Bild- oder Zieldaten, fehlendem Nachweis einer instabilen Fraktur und/oder eines zeitlichen Abstandes der Bildgebung zum Unfallereignis $>48 \mathrm{~h}$ ausgeschlossen werden. Damit verblieben 33 polytraumatisierte bzw. mehrfach-verletzte Patienten mit instabiler BWS-Verletzung, die in die endgültige Studiengruppe aufgenommen wurden. Diese hatten ein Durchschnittsalter von $43,5 \pm 20,1$ Jahren (Spannweite 14-83 Jahre) und waren in 25 Fällen (76\%) männlichen Geschlechts. Die durchschnittliche Hospitalisationsdauer betrug 59,8 $\pm 68,1$ Tage (Spannweite 5-231 Tage), die Mortalitätsrate innerhalb des Studienkollektivs lag bei 21\% (7 Patienten). Zwischen dem Patientengeschlecht und der Mortalitätsrate fand sich ein hoch-signifikanter Zusammenhang im Sinne einer schlechteren Prognose bei Frauen (Mortalitätsrate bei Frauen $75 \%$ bzw. bei Männern 4\%, $p<0,001$ ). Das Patientenalter hatte keinen signifikanten Einfluss auf die Überlebenswahrscheinlichkeit (Durchschnittsalter der Verstorbenen bzw. der Überlebenden: 48,8 \pm 25 ,6 Jahre bzw. 42,0 $\pm 18,8$ Jahre; $p=0,278$ ). 

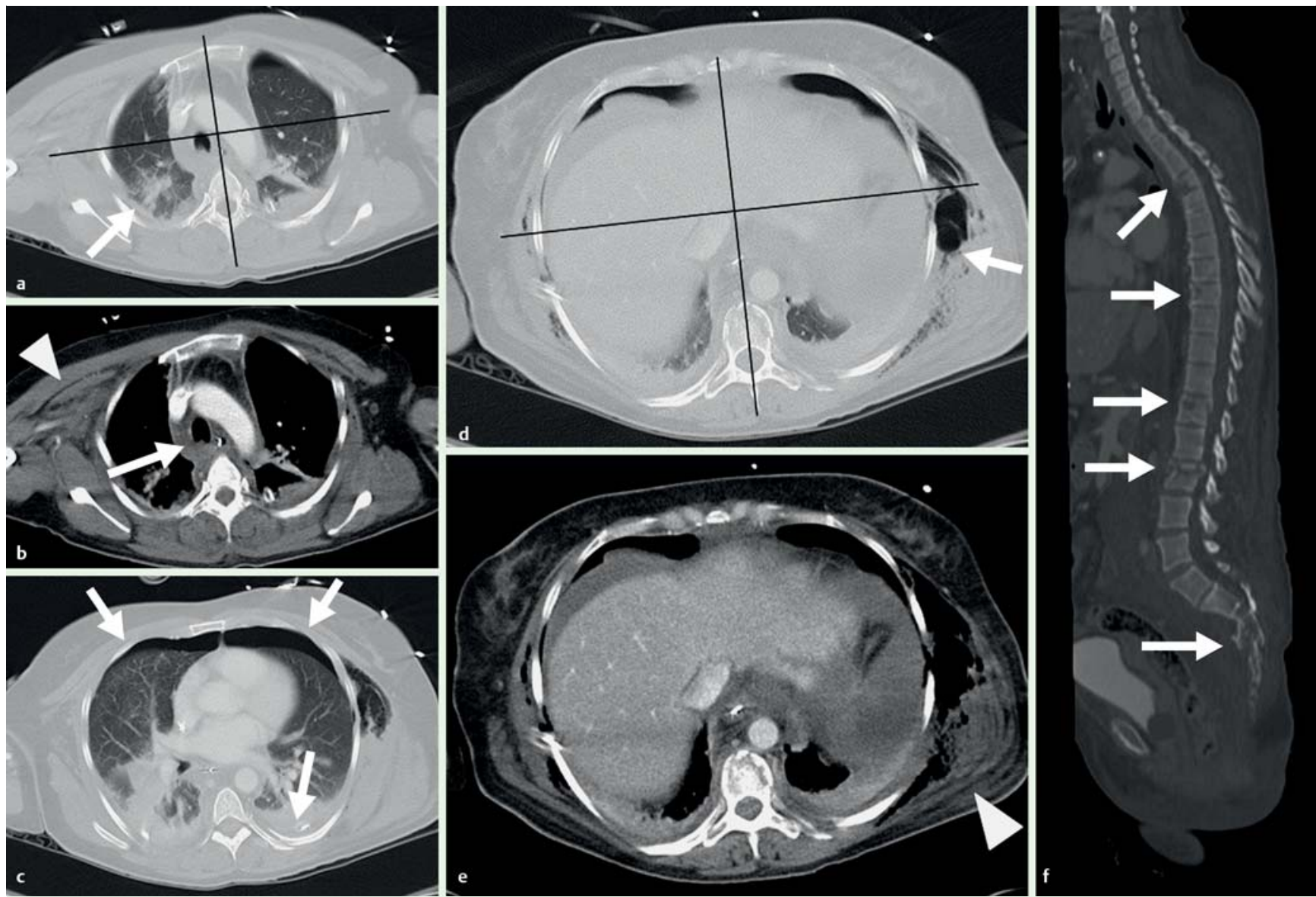
Abb. 1 Verstorbene 42-jährige Frau nach Sturz aus großer Höhe: Lungenkontusionen von $25 \%-50 \%$ auf Höhe des Obergeschosses (a), dorsales mediastina-
les Hämatom (b), Rippenfrakturen und bilaterale Pneumothoraces (c), beidseitige Prellmarken (b/e, weiße Pfeilköpfe) und Weichteilemphysem links $>90^{\circ}$ des thorakalen Umfangs (d), multiple Frakturen von BWK3, BWK8, BWK12, LWK2 und SWK2/3 (f).

\section{Bildauswertung}

Von 33 Patienten zeigten 21 (64\%) Lungenkontusionen, je 13 (39\%) Pleuraergüsse und Wirbelsäulenverletzungen in mehrfachen Lokalisationen, zehn (30\%) Rippenfrakturen, acht (24\%) Pneumothoraces und fünf $(15 \%)$ Weichteilverletzungen (siehe - Abb. 1 u. 2).

Clavicula- und Scapulafrakturen bzw. Dissektionen/Rupturen der Aorta, der supraaortalen Arterien oder der V. cava superior wurden nicht beobachtet. $\odot$ Tab. 1 illustriert die absoluten und relativen Häufigkeiten der beobachteten thorakalen Verletzungen innerhalb des Studienkollektivs.

Der Kontusions-Score lag für alle Patienten im Median bei 1 ( 0 12), wobei die verstorbenen Patienten einen signifikant höheren medianen Scorewert aufwiesen (4,0 Punkte [0 - 12 Punkte] bei Verstorbenen versus 1,0 Punkte [0-10 Punkte] bei Überlebenden; $p=0,016]$ ).

Folgende CT-Befunde waren in der explorativen Chi-QuadratTestung signifikant mit der Mortalität assoziiert: Prellmarken des Thorax mit Weichteilemphysem $>90^{\circ}$ des thorakalen Umfangs $(p=0,006)$, Rippenfrakturen $(p=0,016)$, mediastinale Hämatome $(p=0,027)$, bilaterale Pneumothoraces von 1 bis $3 \mathrm{~cm}$ Saumbreite $(p=0,040)$, bilaterale Lungenkontusionen/-einblutungen auf Untergeschosshöhe mit $25-50 \%$ der Fläche auf gleicher Höhe $(p=0,023)$ und das Vorhandensein bilateraler Thoraxdrainagen $(p=0,040)$. In der multivariaten Analyse nach Korrektur der Variablenabhängigkeit untereinander verblieben eine signifikante Abhängigkeit der Mortalität für thorakale Prellmarken mit Weichteilemphysem $>90^{\circ}$, Rippenfrakturen, mediastinale Hämatome und bilaterale Pneumothoraces mit einer Saumbreite von $1-3 \mathrm{~cm}$. $\odot$ Tab. 2 demonstriert die Aussagekraft der multivariat als signifikant verbliebenen Variablen in Bezug auf die Mortalität.

\section{Diskussion}

\section{$\nabla$}

Die initiale Einschätzung des polytraumatisierten Patienten bezüglich der Verletzungsschwere, Therapiebedürftigkeit und Prognose ist eine schwierige Aufgabe, zumal diese unter Zeitdruck innerhalb der ersten 60 Minuten nach Trauma erfolgen sollte [3]. Der modernen Schnittbildgebung kommt hierbei eine entscheidende Rolle zu, da im Rahmen der initialen MDCT-Diagnostik des Polytraumatisierten in rascher Zeit ausgedehnte Körperabschnitte untersucht sowie große Bilddatenmengen berechnet und ausgewertet werden können. Die präzise Gestaltung des Ablaufes der bildgebenden Diagnostik, der Scan-/RekonstruktionsParameter und schließlich der adäquaten Bilddokumentation bzw. -präsentation sind Gegenstand der wissenschaftlichen Diskussion [15]. Zum gegenwärtigen Zeitpunkt sind für den technischen Ablauf der Bildgebung weder von Seiten der Deutschen Röntgengesellschaft noch der Deutschen Gesellschaft für Unfallchirurgie detaillierte Leitlinien verfügbar. Unbestritten ist jedoch die Überlegenheit der MDCT nicht nur bei der Diagnostik von Wirbelsäulen-Verletzungen [11-15], sondern auch bei der Ab- 

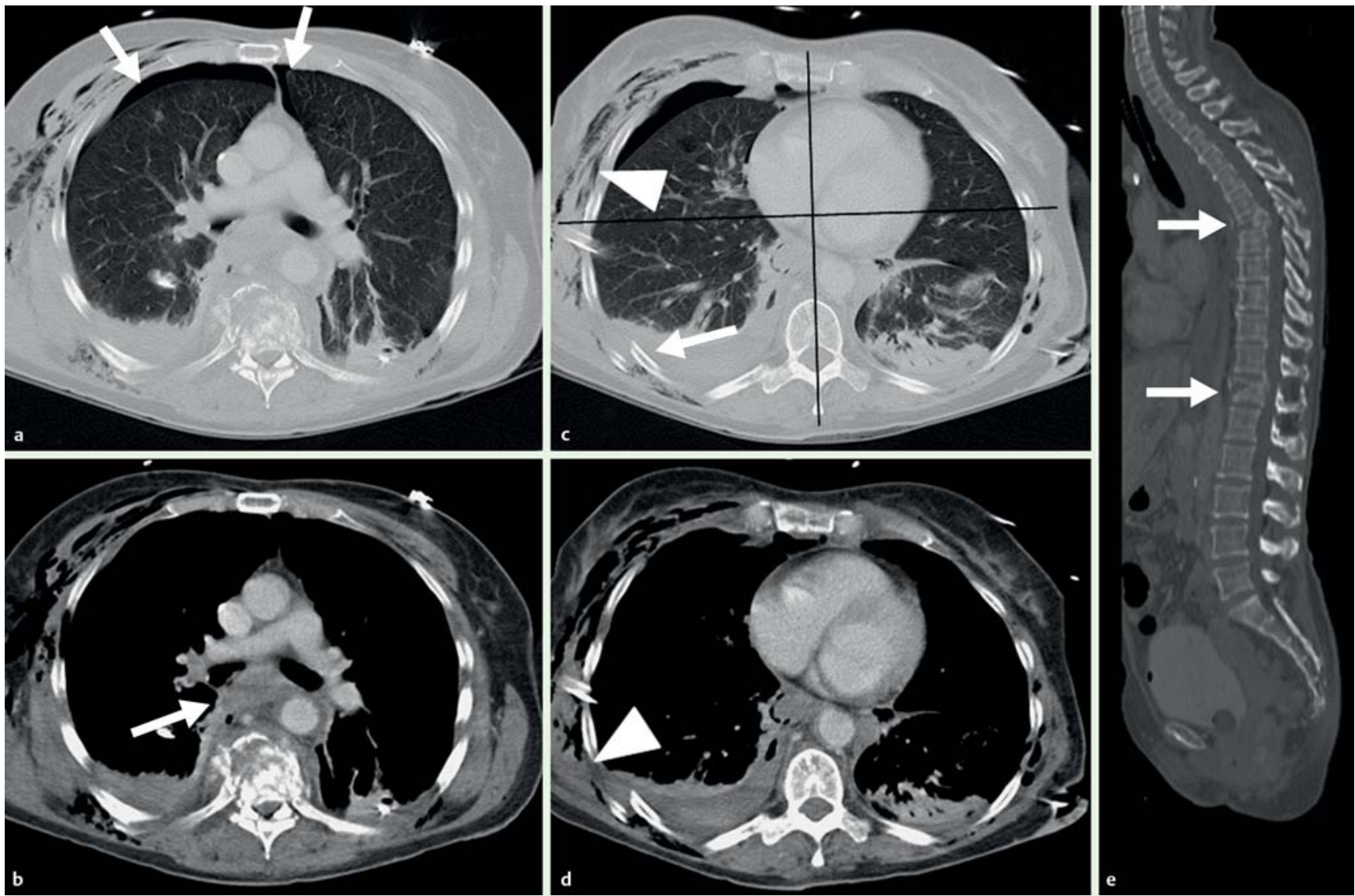

Abb. 2 Verstorbene 53-jährige Frau mit BWK6- und BWK12-Fraktur (e): Bilaterale Pneumothoraces (a), mediastinales Hämatom (b), Rippenfrakturen (c) und Prellmarke mit Weichteilemphysem $>90^{\circ}$ des thorakalen Umfangs (c/d, weiße Pfeilköpfe).

klärung von Verletzten mit thorakalem Trauma [20,21]. Der häufigere Einsatz der MDCT basiert neben den o.g. eindrücklichen Fortschritten in der Gerätetechnik auch auf Weiterentwicklungen in der Nachverarbeitung des Bildmateriales, welche eine nahezu beliebig wählbare Form der Bilddarstellung ermöglicht: So kann aus einem thorakalen Scanvorgang sowohl das intrathorakale Volumen als auch die BWS in sämtlichen Raumebenen berechnet werden. Dies ermöglicht der MDCT-Diagnostik durch die gleichzeitige Erfassung mehrerer Organsysteme eine elegante Entsprechung im Hinblick auf den Zeitdruck, fordert vom Untersucher aber auch eine ganzheitliche, organübergreifende Sicht der akquirierten Bilddaten zur Evaluation möglicher zusätzlicher pulmonaler und thorakaler Verletzungen beim BWS-Trauma. Der Auswahl bzw. Einrichtung von geeigneten Scan- und Nachverarbeitungsalgorithmen kommt damit eine hohe Bedeutung zu.

Die hier in der multivariaten Analyse nach Korrektur der Variablenabhängigkeit als signifikant verbliebenen Befunde entsprechen von ihrer Qualität her den Folgen eines erheblichen Thoraxtraumas: Korrespondierend zu unserer Analyse von Rippenfrakturen als signifikantem Befund konnten Liman et al. nachweisen, dass das Vorliegen von zwei oder mehr gebrochenen Rippen beim Thoraxtrauma (neben einem Patientenalter $>60$ Jahre und einem Injury-Severity-Score > 16) mit einer erhöhten Mortalität assoziiert war [22]. Auch in der Publikation von Sirmali et al. konnte eine Korrelation zwischen der Anzahl von Rippenfrakturen und der Mortalitätsrate nachgewiesen werden [23]. Gleiches zeigten Bulger et al. auch beim älteren Patienten, wobei ältere Patienten $\geq 65$ Jahre mit Thoraxtrauma und Rippenfrakturen eine zweifach höhere Mortalitäts- und thorakale Morbiditätsrate im
Vergleich zu jüngeren Patienten mit gleichen Verletzungen aufwiesen [24]. Flagel et al. wiesen ebenfalls eine Assoziation von pulmonal bedingter Morbidität bzw. Mortalität und ansteigender Anzahl von gebrochenen Rippen nach [25]. Rippenbrüchen kommt damit eine prognostische Bedeutung zu; eine detaillierte Evaluation des Bildmateriales diesbezüglich ist daher notwendig. Bislang ist wenig zur Wertigkeit von Weichteilläsionen bei Thoraxverletzungen bekannt. Die in unserer Studie als signifikant mit der Mortalität assoziierten Prellmarken mit subkutanem Emphysem wurden bislang nicht als prognostischer Bildbefund beschrieben. Allerdings wurde ein subkutanes Weichteilemphysem in Kombination mit anderen Faktoren wie Rippenfrakturen, weiblichem Geschlecht und Lungenkontusionen in einer Studie von Ball et al. als unabhängiger prognostischer Faktor für okkulte Pneumothoraces identifiziert [26]. Im Gegensatz zu Athanassiadi et al. konnten wir eine signifikante Assoziation von bilateralen Pneumothoraces mit der Mortalität nachweisen: In ihrer Studie beeinflusste das Vorliegen von Pneumo- oder Hämatothoraces bei Patienten mit sog. „Flail-Chest“ im Sinne eines instabilen Thorax zwar nicht die Mortalität, wohl aber die Hospitalisationsdauer [27]. Auch Virgos et al. fanden keine prognostische Bedeutung von Hämato- oder Pneumothoraces bei 108 Patienten mit Thoraxtrauma; hier waren als pneumologische Faktoren die Beatmungspflichtigkeit, ein hoher positiver end-exspiratorischer Druck und Lungenkontusionen signifikant mit dem Versterben verbunden [28]. Die prognostische Wertigkeit von bildmorphologisch nachgewiesenen Lungenkontusionen ist umstritten: Während die überwiegende Mehrzahl der Studien zu Lungenkontusionen eine Assoziation mit der Mortalität [28], Pneumo- 
Tab. 1 Beobachtete thorakale Traumafolgen bei 33 Patienten mit instabiler BWS-Verletzung.

$\begin{array}{lc}\text { Betroffene Thoraxregion } & \mathbf{n}(\%) \\ \text { Lunge/Pleura } & \\ \text { - dorsale, lagebedingte Minderbelüftungen } & 25(76) \\ \text { - Atelektasen } & 8(24) \\ \text { bilaterale Atelektasen } & 4(12) \\ \text { - Pneumothorax } & 8(24) \\ \text { einseitiger Pneumothorax, Saumbreite bis } 1 \text { cm } & 3(9) \\ \text { einseitiger Pneumothorax, Saumbreite } 1 \text { bis 3 cm } & 3(9) \\ \text { beidseitiger Pneumothorax, Saumbreite } 1 \text { bis 3 cm } & 2(6) \\ \text { Pneumothorax, Saumbreite mehr als 3 cm } & 1(3) \\ \text { - Lungenkontusion/-einblutung } & 21(64) \\ \text { Kontusions-Score 0 - 6 Punkte } & 18(55) \\ \text { Kontusions-Score 7 - 12 Punkte } & 3(9) \\ \text { - Lungenlazeration } & 2(6) \\ \text { - Pleuraerguss } & 13(39) \\ \text { - einliegende Thoraxdrainage(n) } & 4(12) \\ \text { Mediastinum } & \\ \text { - mediastinales Hämatom } & 11(33) \\ \text { mediastinales Hämatom, Größe bis } 5 \mathrm{~cm} & 7(21) \\ \text { mediastinales Hämatom, Größe über } 5 \mathrm{~cm} & 4(12) \\ \text { - aktive mediastinale Blutung mit KM-Austritt } & 1(3) \\ \text { - Verletzungen von Aorta und/oder V. cava superior } & 0 \\ \text { Thoraxwand } & \end{array}$

nien [29] und dem Auftreten eines ARDS [30] nachweisen konnten, ließ sich in einzelnen Publikationen [31] keine prognostische Wertigkeit von isolierten Lungenkontusionen ermitteln. Zwar waren in unserem Kollektiv Lungenkontusionen in der multivariaten Analyse nicht signifikant assoziiert, dennoch wiesen die Verstorbenen einen signifikant höheren Kontusionsscore auf. Eine mögliche Erklärung unserer Ergebnisse liegt in dem Einschlusskriterium des zeitlichen Abstandes zum Unfallereignis: Pape et al. wiesen zwar eine engere Assoziation von konventionell-radiologisch detektierten Verletzungen des Lungenparenchyms im Vergleich zu Thoraxwand-Verletzungen im Hinblick auf ein ungünstiges Outcome nach, erstere waren aber oft erst nach 24 Stunden nachweisbar [32]. Auch bei Tyburski et al. zeigte sich bei 26 von 103 Patienten mit Thoraxtrauma, dass ein Progress initialer, konventionell radiologisch fassbarer Lungenkontusionen mit einer höheren Mortalität verbunden war [33]. Diese Ergebnisse deuten auf eine Zeitabhängigkeit des Nachweises von Lungenkontusionen hin, sodass unsere Ergebnisse hierdurch beeinflusst worden sein könnten.
Tab.2 Prognostische Aussagekraft der signifikanten Variablen bezüglich der Mortalität.

\begin{tabular}{|c|c|c|c|c|c|}
\hline Variable & SE & SP & PPW & NPW & $\mathrm{OA}$ \\
\hline $\begin{array}{l}\text { Prellmarke mit Weichteil- } \\
\text { emphysem }>90^{\circ}\end{array}$ & 43 & 100 & 100 & 87 & 88 \\
\hline Rippenfrakturen & 71 & 81 & 50 & 91 & 79 \\
\hline mediastinales Hämatom & 71 & 77 & 45 & 91 & 76 \\
\hline $\begin{array}{l}\text { bilaterale Pneumothoraces } \\
\text { mit } 1-3 \mathrm{~cm} \text { Saumbreite }\end{array}$ & 29 & 100 & 100 & 84 & 85 \\
\hline
\end{tabular}

Interessanterweise zeigte sich - entgegen anderen Publikationen - kein Zusammenhang zwischen einem höheren Alter und der Mortalität, was möglicherweise durch das relativ junge Durchschnittsalter unserer Patientengruppe bedingt ist. Schließlich fanden wir eine hochsignifikante Assoziation von weiblichem Geschlecht und Mortalität. Eine gleichartige Konstellation zu ungunsten von Frauen ließ sich interessanterweise bei Ball et al. [26] in Bezug auf okkulte Pneumothoraces beobachten, wobei bilaterale Pneumothoraces in unserer Studie prädiktiv für die Mortalität waren. Wang et al. [34] konnten allerdings bei $127 \mathrm{~Pa}$ tienten mit thorakalem Trauma und Intensivpflichtigkeit keine Unterschiede zwischen Überlebenden und Verstorbenen im Hinblick auf das Geschlecht feststellen. Dem gegenüber zeigten Altenbernd et al. [35] bei wirbelverletzten Patienten mit Spondylitis ankylosans eine signifikante Korrelation von weiblichem Geschlecht und Sterberate. Eine mögliche Erklärung für einen Einfluss des Geschlechtes bei wirbelsäulenverletzten Patienten kann das häufigere Vorliegen einer Osteoporose bei Frauen sein, wobei ein erhöhtes Risiko einer Verletzung bei Osteoporose lediglich für die Wirbelsäule, aber nicht anderen Körperregionen bei Verkehrsunfällen dokumentiert werden konnte. Letztlich verbleibt die genaue Wertigkeit zwischen der Assoziation von weiblichem Geschlecht und Mortalität angesichts der niedrigen Fallzahl und des retrospektiven Studiencharakters unklar.

Unabhängig von der Diskussion um einzelne prognostische Bildbefunde zeigen unsere Ergebnisse, dass eine orientierende prognostische Einschätzung des Verletzten in Bezug auf das stattgehabte Thoraxtrauma anhand einer Evaluation von Rippenfrakturen, mediastinalem Hämatom, Weichteilemphysemen und bilateralen Pneumothoraces möglich ist. Die Versorgung dieser komplex verletzten Patienten obliegt initial sicher zunächst dem Bereich der Unfallchirurgie in interdisziplinärer Zusammenarbeit mit den Disziplinen der Anästhesie und der Thoraxchirurgie. Dennoch kann der oft komplexe Verlauf eines schwer Thoraxverletzten eine frühzeitige Einbeziehung und engen fachübergreifenden Austausch aller Disziplinen erfordern, da die konservative begleitende Therapie einen erheblichen - im Fall des Thoraxverletzten entscheidenden - Einfluss auf die Prognose hat. Der additiven internistischen bzw. pneumologischen Betreuung und Beurteilung dieser Patientengruppe kommt daher ebenso eine Bedeutung zu, sodass ein rasches Erkennen prognostischer CT-Bildbefunde bei BWS- bzw. konsekutiv Thorax-Verletzten auch für primär nicht-operative Disziplinen von Wert ist. Inwiefern hierdurch die pulmonal bedingte Morbidität und Mortalität dieser Patientengruppe positiv beeinflusst werden kann, bleibt allerdings weiteren Studien vorbehalten.

Unsere Studie weist folgende Limitationen auf: Zunächst erfolgte die statistische Auswertung zur Identifikation von möglichen prognostischen Risikofaktoren aufgrund der repetitiven Testung 
des Kollektives explorativ mit höherer Irrtumswahrscheinlichkeit. In der im Anschluss durchgeführten multivariaten Analyse verblieben lediglich die o.g. Faktoren als prognostische Befunde. Zudem ist angesichts des retrospektiven Studiencharakters ein Selektions-Bias der Patienten - auch angesichts der niedrigen Patientenzahl - prinzipiell nicht auszuschließen. Als Haus der Maximalversorgung mit Schwerpunkt in der Unfall- und Wirbelsäulenchirurgie ist dennoch von einer erhöhten Zuweisung dieser Patientengruppe auszugehen. Zudem sind angesichts der relativ streng gewählten Einschlusskriterien höhere Patientenzahlen nur schwierig zu erreichen. Insbesondere das Kriterium der instabilen Fraktur hat die Patientengruppe stark selektiert. Diesem Umstand lag die Vorstellung zugrunde, dass eine stabile bzw. geringfügige Fraktur keine hinreichende Kraftwirkung auf den Thorax bedingt. Die beschriebenen Ergebnisse sind damit möglicherweise nicht voll repräsentativ für alle Polytraumatisierten mit BWS-Verletzung.

Zusammenfassend ließen sich in unserer Studiengruppe von polytraumatisierten bzw. mehrfach-verletzten Patienten mit instabiler BWS-Fraktur prognostische Bildbefunde identifizieren. Die MDCT-Diagnostik bei betroffenen Patienten muss damit in der Erfassung und Auswertung der Bilddaten sowohl der BWS-Abklärung als auch der Evaluation von Thorax und Lunge Rechnung tragen.

\section{Interessenkonflikte}

$\nabla$

Die Autoren geben an, dass kein Interessenkonflikt besteht.

\section{Institute}

Institut für Diagnostische Radiologie, Interventionelle Radiologie und Nuklearmedizin (Direktor: Prof. Dr. V. Nicolas), Berufgenossenschaftliches Universitätsklinikum Bergmannsheil GmbH, Ruhr-Universität Bochum Chirurgische Klinik und Poliklinik (Direktor: Prof. Dr. T.A. Schildhauer), Berufgenossenschaftliches Universitätsklinikum Bergmannsheil GmbH, Ruhr-Universität Bochum

3 Medizinische Klinik III, Klinik für Pneumologie, Allergologie und Schlaf- und Beatmungsmedizin (Komm. Leiter: Prof. Dr. A. Mügge), Berufgenossenschaftliches Universitätsklinikum Bergmannsheil GmbH, Ruhr-Universität Bochum

\section{Literatur}

1 Statistisches Bundesamt. Todesursachenstatistik 2009. In: GesundheitTodesursachen in Deutschland. Statistisches Bundesamt; 2011; Artikelnummer 2120400097004

2 Khandhar SJ, Johnson SB, Calhoon JH. Overview of thoracic trauma in the United States. Thorac Surg Clin 2007; 17: 1 -9

3 McNicholl BP. The golden hour and prehospital trauma care. Injury 1994; 25: 251 - 254

4 Davis JW, Hoyt DB, McArdle MS et al. An analysis of errors causing morbidity and mortality in a trauma system: a guide for quality improvement. J Trauma 1992; 32: 660 - 665; discussion 665-666

5 Davis JW, Hoyt DB, McArdle MS et al. The significance of critical care errors in causing preventable death in trauma patients in a trauma system. J Trauma 1991; 31: 813-818; discussion 818-819

6 Lemburg SP, Peters SA, Roggenland D et al. Cumulative effective doses of diagnostic imaging procedures in adolescents with spinal injuries. AJR Am J Roentgenol 2010; 195: 1411-1417

7 Salottolo K, Bar-Or R, Fleishman $M$ et al. Current utilization and radiation dose from computed tomography in patients with trauma. Crit Care Med 2009; 37: 1336-1340

8 Mayo JR, Aldrich J, Muller NL et al. Radiation exposure at chest CT: a statement of the Fleischner Society. Radiology 2003; 228: 15-21

9 Nickoloff EL, Alderson PO. Radiation exposures to patients from CT: reality, public perception, and policy. AJR Am J Roentgenol 2001; 177 : $285-287$
10 Brenner DJ, Hall EJ. Computed tomography - an increasing source of radiation exposure. N Engl J Med 2007; 357: 2277-2284

11 Diaz JJ Jr., Gillman C, Morris JA Jr. et al. Are five-view plain films of the cervical spine unreliable? A prospective evaluation in blunt trauma patients with altered mental status. J Trauma 2003; 55: 658 - 663; discussion $663-654$

12 Mathen $R$, Inaba K, Munera F et al. Prospective evaluation of multislice computed tomography versus plain radiographic cervical spine clearance in trauma patients. J Trauma 2007; 62: 1427 - 1431

13 Mower WR, Hoffman JR, Pollack CV Jr. et al. Use of plain radiography to screen for cervical spine injuries. Ann Emerg Med 2001; 38: 1-7

14 Schenarts PJ, Diaz J, Kaiser C et al. Prospective comparison of admission computed tomographic scan and plain films of the upper cervical spine in trauma patients with altered mental status. J Trauma 2001; 51: 663 -668; discussion 668-669

15 Holmes JF, Akkinepalli $R$. Computed tomography versus plain radiography to screen for cervical spine injury: a meta-analysis. J Trauma 2005; 58: $902-905$

16 Kiraly L, Schreiber M. Management of the crushed chest. Crit Care Med 2010; 38: S469-S477

17 Schmitt J, Midha M, McKenzie N. Medical complications of spinal cord disease. Neurol Clin 1991; 9: 779-795

18 van den Berg ME, Castellote JM, de Pedro-Cuesta J et al. Survival after spinal cord injury: a systematic review. J Neurotrauma 2010; 27: $1517-1528$

19 Denis $F$. The three column spine and its significance in the classification of acute thoracolumbar spinal injuries. Spine 1983; 8: 817-831

20 Grieser T, Bühne KH, Hauser H et al. Relevanz der Befunde von Thoraxröntgen und Thorax-CT im routinemäßigen Schockraumeinsatz bei 102 polytraumatisierten Patienten - eine prospektive Studie. Fortschr Röntgenstr 2001; 173: 44-51

21 Trupka A, Waydhas C, Hallfeldt KK et al. Value of thoracic computed tomography in the first assessment of severely injured patients with blunt chest trauma: results of a prospective study. J Trauma 1997; 43: 405-411; discussion 411-402

22 Liman ST, Kuzucu A, Tastepe AI et al. Chest injury due to blunt trauma. Eur J Cardiothorac Surg 2003; 23: $374-378$

23 Sirmali M, Turut H, Topcu S et al. A comprehensive analysis of traumatic rib fractures: morbidity, mortality and management. Eur J Cardiothorac Surg 2003; 24: 133-138

24 Bulger EM, Arneson MA, Mock CN et al. Rib fractures in the elderly. J Trauma 2000; 48: 1040-1046; discussion 1046-1047

25 Flagel BT, Luchette FA, Reed RL et al. Half-a-dozen ribs: the breakpoint for mortality. Surgery 2005; 138: 717-723; discussion 723-715

26 Ball CG, Kirkpatrick AW, Laupland KB et al. Incidence, risk factors, and outcomes for occult pneumothoraces in victims of major trauma. J Trauma 2005; 59: 917-924; discussion 924-915

27 Athanassiadi K, Gerazounis M, Theakos N. Management of 150 flail chest injuries: analysis of risk factors affecting outcome. Eur J Cardiothorac Surg 2004; 26: 373 - 376

28 Virgos Senor B, Nebra Puertas AC, Sanchez Polo C et al. Predictors of outcome in blunt chest trauma. Arch Bronconeumol 2004; 40: 489-494

29 Allen GS, Coates NE. Pulmonary contusion: a collective review. Am Surg 1996; 62: 895-900

30 Miller PR, Croce MA, Bee TK et al. ARDS after pulmonary contusion: accurate measurement of contusion volume identifies high-risk patients. J Trauma 2001; 51: 223 -228; discussion 229-230

31 Hoff SJ, Shotts SD, Eddy VA et al. Outcome of isolated pulmonary contusion in blunt trauma patients. Am Surg 1994; 60: 138-142

32 Pape HC, Remmers D, Rice J et al. Appraisal of early evaluation of blunt chest trauma: development of a standardized scoring system for initial clinical decision making. J Trauma 2000; 49: 496-504

33 Tyburski JG, Collinge JD, Wilson RF et al. Pulmonary contusions: quantifying the lesions on chest X-ray films and the factors affecting prognosis. J Trauma 1999; 46: 833 - 838

34 Wang SH, Wei TS, Chen CP. Prognostic analysis of patients with blunt chest trauma admitted to an intensive care unit. J Formos Med Assoc 2007; 106: $444-451$

35 Altenbernd J, Bitu S, Lemburg S et al. Wirbelfrakturen bei Patienten mit Spondylitis ankylosans: Eine retrospektive Analyse von 66 Patienten. Fortschr Röntgenstr 2009; 181: 45 - 53

36 Rupp JD, Flannagan CA, Hoff CN et al. Effects of osteoporosis on AIS 3+ injury risk in motor-vehicle crashes. Accid Anal Prev 2010; 42: 2140 2143 